\title{
Combined X-Ray and Fully Leaky Guided Mode Studies of the Smectic Layer and Optic Tensor Configuration in a Ferroelectric Liquid-Crystal Cell
}

\author{
B. Hodder* and J. R. Sambles \\ Thin Film Photonics, School of Physics, University of Exeter, Stocker Road, Exeter EX4 4QL, United Kingdom \\ S. Jenkins and R. M. Richardson ${ }^{\dagger}$ \\ Liquid Crystals Group, Chemistry Department, University of Bristol, Cantocks Close, Cotham, Bristol BS8 ITS, United Kingdom \\ (Received 17 December 1999)
}

\begin{abstract}
X-ray scattering together with optical characterization using fully leaky guided modes have been used for the first time to study the same ferroelectric liquid-crystal cell. This enables direct calculation of an accurate cone and chevron description of the liquid-crystal director profile since the layer structure and optic tensor configuration are both well known.
\end{abstract}

PACS numbers: 61.30.Eb, 42.70.Df, 77.84.Nh

Ferroelectric liquid-crystal (FLC) devices are primarily of interest owing to their truly bistable operation and fast switching times, in the $\mu$ s regime [1]. These characteristics are ideally suited to low power optical display devices. In an unconstrained or "free" sample of smectic FLC material the molecules are arranged to form a density wave of material. Usually, the density wave description is simplified by making the assumption that the molecules are arranged in well-defined layers, referred to as smectic layers. Typical commercially available FLC materials undergo a second order smectic $A\left(S_{A}\right) \Leftrightarrow$ chiral smectic $C\left(S_{C}^{*}\right)$ phase transition (where the symbol $(*)$ denotes chirality). In each phase the molecular alignment is characterized by the primary director that defines the average orientation of the molecular long axes. At high temperatures, in the $S_{A}$ phase, the primary director orientation is perpendicular to the smectic layers (parallel to the density wave vector). At lower temperatures, in the $S_{C}^{*}$ phase, the primary director tilts away from the smectic layer normal [2]. The tilt plane is not biased in any given direction and there exists an infinity of possible primary director orientations that specify a cone of allowed positions. Additionally, the director precesses in a helical fashion through the sample about an axis perpendicular to the smectic layers. Also, the $C_{2}$ optical symmetry of the biaxial $S_{C}^{*}$ phase supports a spontaneous transverse polarization $\left(P_{S}\right)$ which is largely responsible for the fast switching times achieved using surface stabilized ferroelectric liquid-crystal (SSFLC) devices.

The optical tensor configuration and smectic layer behavior in SSFLC devices are rather different from that of the unconstrained sample, and for thin cells (less than a few $\mu \mathrm{m})$ the helical precession of the director is suppressed. Surface alignment in a typical SSFLC device is provided by rubbed polyimide alignment layers that induce homogeneous or planar alignment at the cell walls. Results of early x-ray studies by Reiker et al. [3] suggested that the smectic layers in such devices are tilted with respect to the cell walls in a chevron arrangement. However, the information provided by $\mathrm{x}$-ray scattering experiments pertains only to the smectic layer orientation and cannot be used to investigate the optic tensor configuration that dictates the important optical response of the cell.

Realization of the tilted smectic layer arrangement led to the cone and chevron model, shown in Fig. 1, used to describe the director profile configurations in, and operation of, SSFLC devices [4]. Early studies of the optic tensor configuration took the form of polarized microscopy [5]. It should be appreciated that the results of polarized optical microscopy are severely limited, as they are deduced from an integrated optical response, and cannot be used to spatially resolve the director profile through the cell. To deduce the director profile through a cell requires the use of a nonintegrating optical technique. Interrogation of guided optical modes provides such a technique and is ideally suited to provide details of the director profile in SSFLC cells. Elston et al. were the first to characterize

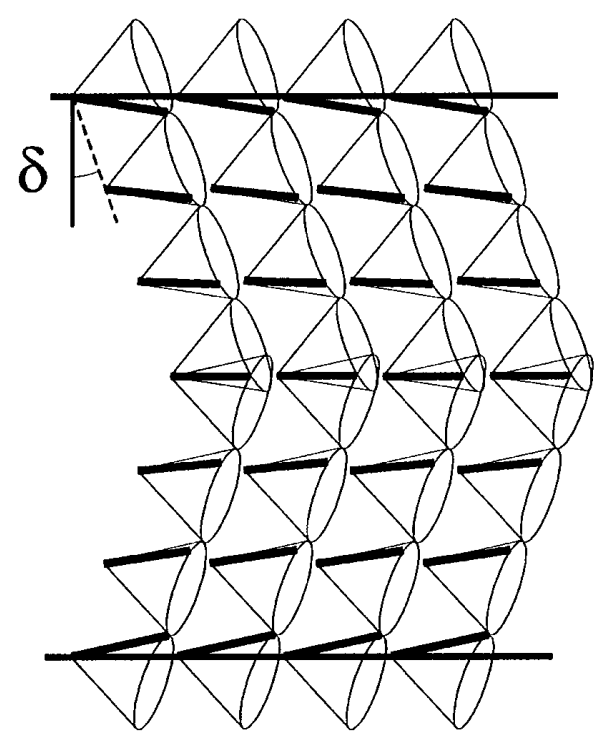

FIG. 1. Illustration of the cone and chevron model used to describe the optic tensor configuration used in a SSFLC device. 
the optic tensor configuration in SSFLCs using such a technique [6]. Their results revealed strong pinning of the primary director both at the surface boundary and at the chevron interface, and provided further evidence for the cone and chevron model. However, the fully guiding technique originally used by Elston et al. is not ideally suited to studies of polyimide aligned SSFLCs as the thin silver layers used to create the optical cavity are damaged by the mechanical rubbing process used to texture the polyimide. Further development in optical waveguide probing of liquid crystal cells led to the fully leaky technique [7] (with high index cells) and later the half-leaky technique [8] which enabled the director profile in SSFLC cells with the conventional polyimide alignment layers to be studied in detail.

It is essential to note that while the guided mode techniques yield the optic tensor configuration in a cell they are not directly sensitive to the orientation of smectic layers. As a result it is not possible to deduce the cone and chevron parameters directly from the Euler twist and tilt angle description of the director profile deduced through fitting guided mode data. Previously, workers calculated the cone and chevron parameters for an assumed cone angle [9]. However, the cone and chevron model derived in this fashion is only as accurate as the assumed cone angle which is often deduced from switching experiments with high voltages applied across the cells. In order to be confident of the cone and chevron parameters it is necessary to combine layer tilt angle measurements obtained through $\mathrm{x}$-ray scattering rocking curve experiments with the results of guided mode studies for the same cell. In this study the latest fully leaky geometry [10], which enables the director profile in cells with standard refractive index glass plates (commercial geometry) to be studied, is used in conjunction with short wavelength $\mathrm{x}$-ray diffraction to characterize the same cell.

The cell geometry employed is illustrated in Fig. 2. Note that the cell [supplied by the Defence and Evaluation Research Agency (DERA), Malvern] is fabricated using 1-mm-thick standard index glass plates used in standard devices. The small angle $\mathrm{x}$-ray scattering experiment at the European Synchrotron Radiation Facility (ESRF) enables studies at an X-ray wavelength of $0.0756 \mathrm{~nm}$. This is only weakly attenuated by glass, enabling the exploration of cells fabricated using thick glass. [Usually, thinner glass plates $(100 \mu \mathrm{m})$ are required for X-ray studies of such devices since glass strongly attenuates the longer wavelength $\mathrm{x}$ rays normally utilized. The thin glass in such cells tends to bow, making them unsuitable for guided mode studies.] Both glass plates are ITO coated and have rubbed polyimide alignment layers that are arranged to give parallel homogeneous alignment of the director at the surface boundaries of the cell. Between the glass and ITO electrodes are $\mathrm{SiO}_{x}$ buffer layers designed to reduce ion injection. Cell thickness and flatness are set using a UV-cured glue that contains $3-\mu \mathrm{m}$-diameter glass spacer beads. The

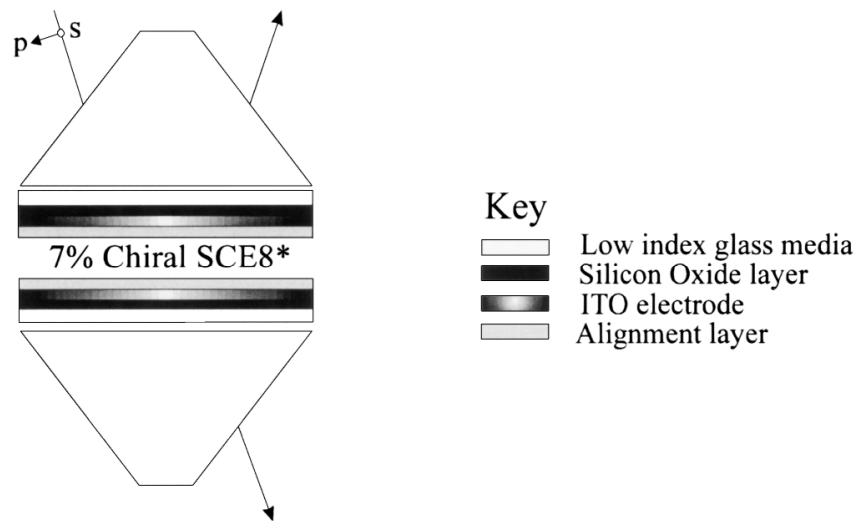

FIG. 2. The cell geometry used in the fully leaky guided mode technique.

cell is filled with a 7\% chiral mixture of SCE8* under vacuum while in the high temperature isotropic phase. It is then cooled at a rate of $4{ }^{\circ} \mathrm{C}$ per hour into the $S_{C}^{*}$ phase. The filled cell can then be studied directly by $\mathrm{x}$-ray scattering, or it may be index matched to the low refractive index entrance and exit prisms of the fully leaky geometry to be studied optically.

$\mathrm{X}$-ray studies of the cell reveal a smectic layer-normal distribution with two peaks which are characteristic of the chevron structure. Both peaks (shown in Fig. 3) are of similar intensity which is indicative of a highly symmetric chevron structure with the chevron interface located centrally within the cell. Extrapolation of a Landau-de Gennes fit to the smectic layer tilt angle as a function of temperature reveals that, at a temperature at $17.0^{\circ} \mathrm{C}$, the smectic layers are tilted at an angle $(\delta)$ of $\pm(23.2 \pm 0.1)^{\circ}$.

Eight data sets are recorded, at a cell temperature of $17.0^{\circ} \mathrm{C}$, using the fully leaky guided mode technique (four reflection, $R_{\mathrm{ss}}, R_{\mathrm{sp}}, R_{\mathrm{ps}}, R_{\mathrm{pp}}$, and four transmission, $T_{\mathrm{ss}}$,

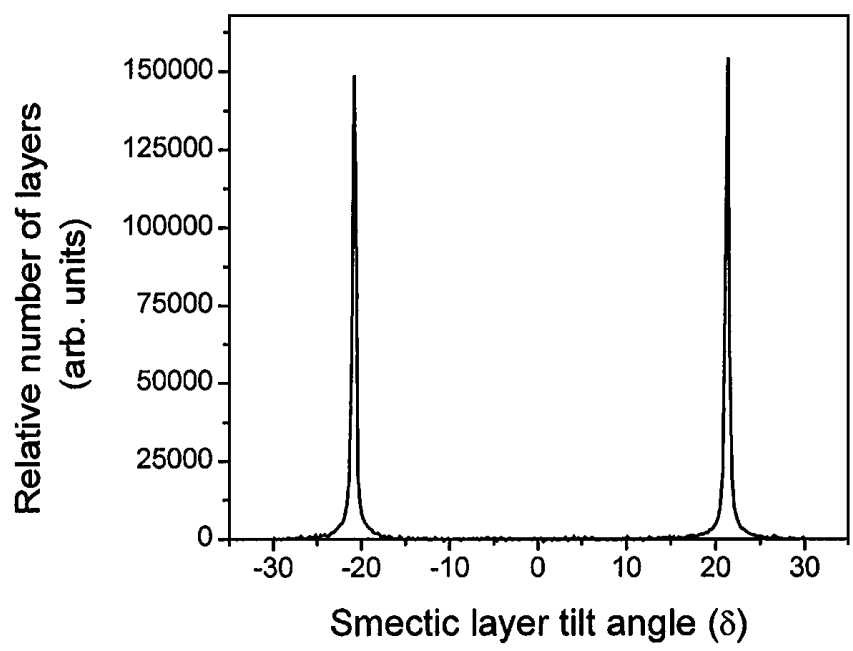

FIG. 3. Smectic layer normal distribution derived from the $\mathrm{x}$-ray Bragg intensities versus layer tilt angle $(\delta)$ at a temperature of $24.5^{\circ} \mathrm{C}$. 


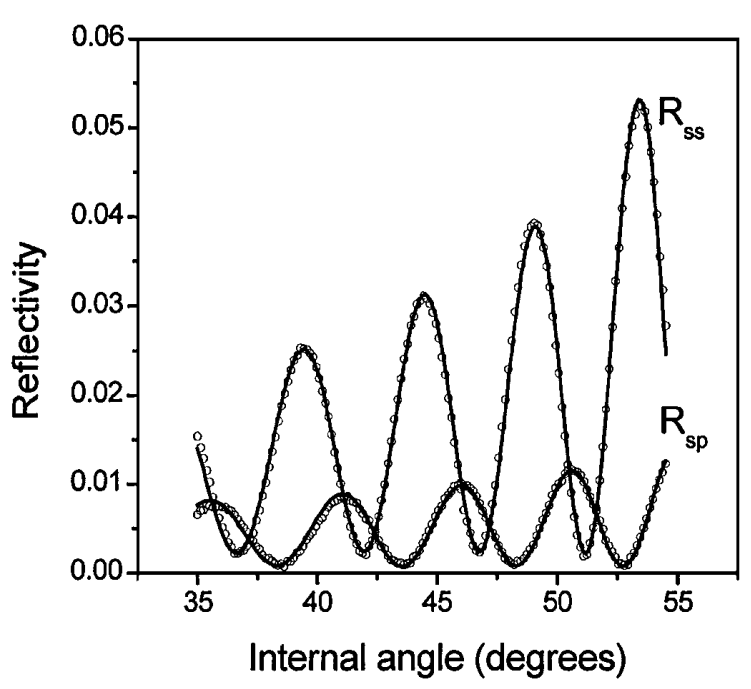

FIG. 4. Fitted guided mode data (1 in 10 points plotted) recorded at an azimuthal setting of $60^{\circ}$.

$T_{\mathrm{sp}}, T_{\mathrm{ps}}, T_{\mathrm{pp}}$, where the subscripts refer to the incident and exit polarizations, respectively). These data sets are fitted together using a single multilayer optics description of the cell parameters and director profile. Illustrative fits (with the cell set at an azimuthal twist angle of $60^{\circ}$ ) are shown in Fig. 4. The Euler twist and tilt angle description of the director profile used to fit the data are shown in Fig. 5. Similarly, high quality fits to angle dependent data recorded at cell azimuths of $0^{\circ}$ and $30^{\circ}$ are also obtained using this model. Multiazimuth fitting provides a rigorous test of the deduced director profile and cell parameters. It is interesting to note the discontinuity in the Euler tilt angle description of the director profile which is attributed to the dispersion in the optic and "mechanical" axes as noted previously by Yang and Sambles [9]. As these authors noted, the structure is strictly biaxial and the only common axis of the biaxial tensor describing the liquid crystal is the $P_{S}$ axis. One might therefore expect the two axes orthogonal to this to disperse with the property studied. Thus there is no reason for the mechanical director axis to coincide with the second optical tensor axis. The optical biaxiality itself is, however, so small that a uniaxial model has been used to fit the optical data.

Equation (1) relates the Euler angles twist $(\phi)$ and tilt $(\theta)$ to the layer tilt $(\delta)$ and cone angle $\left(\theta_{C}\right)$ parameters used in the cone and chevron model. Each of these parameters is defined in Fig. 6. Substituting values of the smectic layer tilt angle and the optical Euler angles determined from the combined $\mathrm{x}$-ray and guided mode experiment yields the cone angle variation represented by the filled circles in Fig. 7:

$$
\cos \left(\theta_{C}\right)=\sin (\phi) \cos (\delta) \sin (\theta)+\sin (\delta) \cos (\theta) .
$$

An alternative interpretation would be to fix the cone angle and to calculate the consequential spread in layer tilt. Substituting a cone angle of $26.2^{\circ}$ into Eq. (1) yields the smectic layer tilt angle variation represented by the open circles

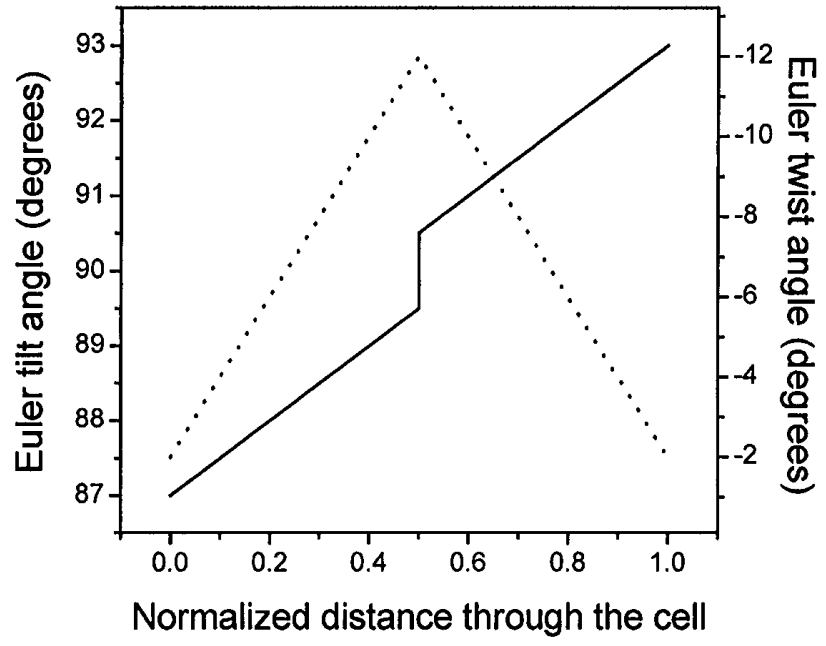

FIG. 5. Euler twist angle (continuous line) and tilt angle (dotted line) descriptions of the liquid-crystal director profile determined through fitting fully leaky data.

in Fig. 7. Both the constant and variable smectic layer tilt configurations yield similar fits to the x-ray peaks owing to the intrinsically broad and symmetric line shape of the smectic layer reflection which results from the onedimensional nature of the smectic order. Thus it is not possible to distinguish, at this finer level, between the two interpretations. However, analysis of the scattering angle of the layer reflection at different points across a peak in the layer-normal distribution data indicates a smooth change in layer spacing of $\sim 0.03 \AA$, thus providing evidence for a cone angle variation of $\sim 0.2^{\circ}$ within the cell. (This is the same order of magnitude as the cone angle variation calculated for a fixed smectic layer tilt angle.) The reality is probably a compromise in which both the cone angle and the smectic layer tilt angle vary by about $0.2^{\circ}$ across the device.

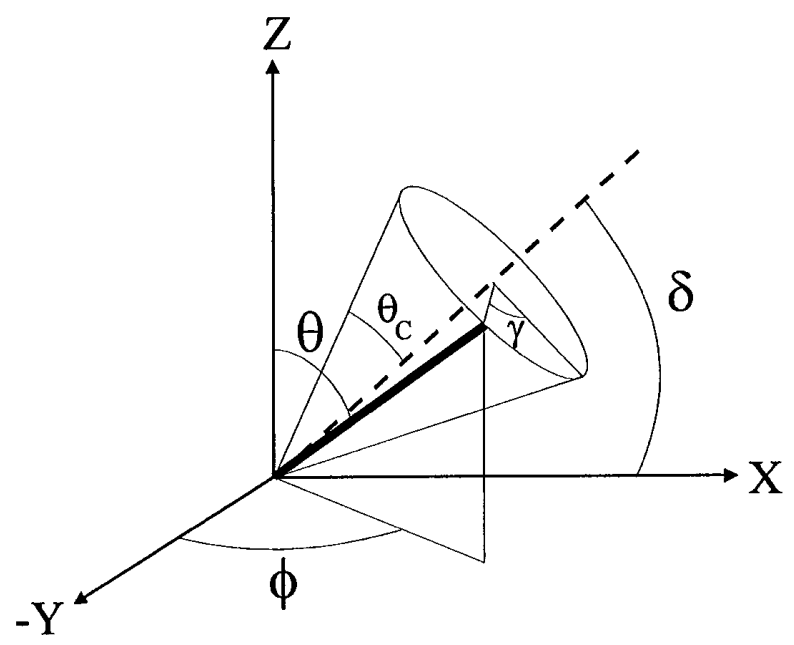

FIG. 6. Definition of the Euler twist angle $(\phi)$, Euler tilt angle $(\theta)$, director rotation angle of the director around the cone $(\gamma)$, smectic layer tilt angle $(\delta)$ and the cone angle $\left(\theta_{C}\right)$. 


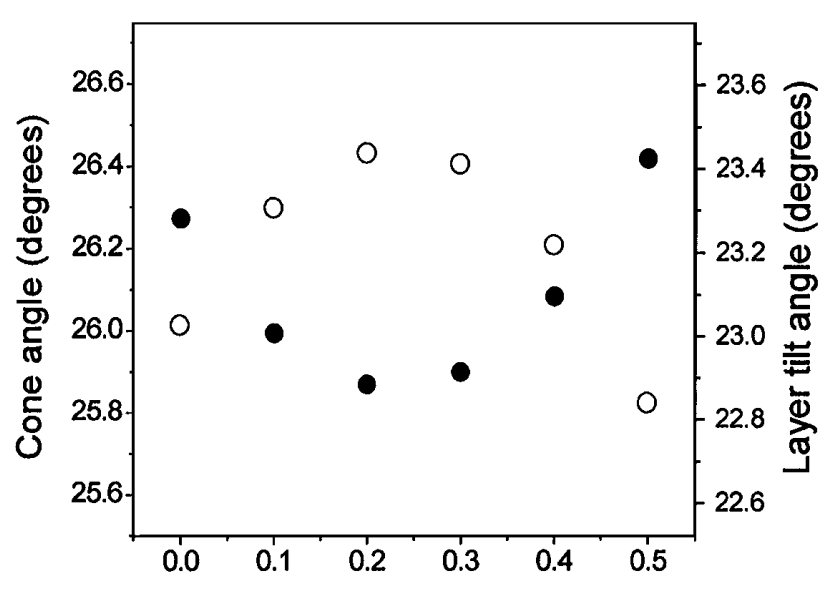

\section{Normalized distance through the cell}

FIG. 7. Cone angle (filled circles) and smectic layer tilt angle variations (open circles) calculated in the upper half of the cell using respective layer tilt angle and cone angle values of $23.2^{\circ}$ and $26.2^{\circ}$.

Substituting values of the cone angle (calculated for a fixed layer tilt angle) and Euler tilt angles into Eq. (2) yields the rotation $(\gamma)$ of the director around the smectic cones [9]:

$$
\cos (\theta)=\cos \left(\theta_{C}\right) \sin (\delta)-\sin \left(\theta_{C}\right) \cos (\delta) \cos (\gamma) .
$$

The rotations (shown in Fig. 8) describe a triangular director profile structure which suggests that the unperturbed $0 \mathrm{~V}$ equilibrium director profile may be described theoretically using a single elastic model. (Note that in the upper half of the cell the rotation is defined from the base of the cone, whereas in the lower half of the cell the rotation is defined from the top of the cone.)

The investigation presented in this Letter provides, for the first time, a complete study of the detailed director profile in a bistable SSFLC device. Combining short wavelength x-ray diffraction from the smectic layers with fully leaky guided wave studies of the optical director of the same cell enables a full cone and chevron model of the director profile to be constructed. The results indicate small elastic deformation in the cone angle near the cell walls and the chevron interface. With the relatively low $P_{S}$ $\left(6.3 \mathrm{nC} \mathrm{cm}^{-2}\right.$ at $\left.20^{\circ} \mathrm{C}\right)$ material used $\left(7 \%\right.$ SCE8 $\left.{ }^{*}\right)$, the director profile shows a simple linear variation of rotation $(\gamma)$ around the cone from the alignment surfaces to the center of the cell. Further studies with this combined technique will be used to explore the voltage switching process

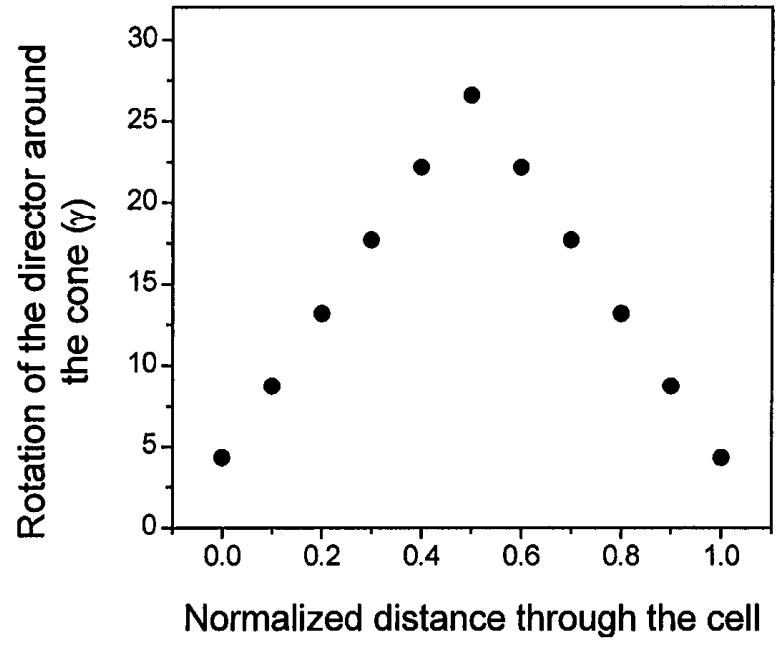

FIG. 8. Description of the SSFLC director configuration in terms of the rotation angle $(\gamma)$ of the director around the set of cones defined by the cone and chevron model parameters calculated in this study.

and the influence of $P_{S}$ on the director profile in standard SSFLC devices.

The authors would like to thank J. M. Petit, T. Narayan (Station ID2, ESRF), DERA (Malvern), and the Engineering and Physical Scientific Research Council.

*Present address: Hewlett-Packard Ltd., Filton Road, Stoke Gifford, Bristol BS34 8QZ, UK.

${ }^{\dagger}$ Present address: H. H. Wills Physics Laboratory, University of Bristol, Tyndall Avenue, Bristol BS8 1TL, UK.

[1] N. A. Clark and S. T. Lagerwall, Appl. Phys. Lett. 36, 899 (1980).

[2] T. R. Taylor, S. L. Arora, and J. L. Fergason, Phys. Rev. Lett. 25, 722 (1970).

[3] T. P. Rieker, N. A. Clark, G. S. Smith, G. S. Parmar, E. B. Sirota, and C. R. Safinya, Phys. Rev. Lett. 59, 2658 (1987).

[4] N. A. Clark and T. P. Rieker, Phys. Rev. A 37, 1053 (1988).

[5] M. A. Handschy and N. A. Clark, Appl. Phys. Lett. 41, 39 (1982).

[6] S. J. Elston, J. R. Sambles, and M. J. Clark, J. Mod. Opt. 36, 1019 (1989).

[7] C. R. Lavers and J.R. Sambles, Ferroelectrics 113, 339 (1991).

[8] F. Yang and J. R. Sambles, J. Opt. Soc. Am. B 10, 5 (1993).

[9] F. Yang and J. R. Sambles, Liq. Cryst. 13, 1 (1993).

[10] F. Yang and J. R. Sambles, J. Opt. Soc. Am. B 16, 3 (1999). 